\title{
Change Detection and Background Extraction by Linear Algebra
}

\author{
EMRULLAH DURUCAN AND TOURADJ EBRAHIMI, MEMBER, IEEE
}

\author{
Invited Paper
}

\begin{abstract}
Change detection plays a very important role in real-time image analysis, e.g., detection of intruders. One key issue is robustness to varying illumination conditions. We propose two techniques for change detection that have been developed to deal with variations in illumination and background, with real-time capabilities. The foundations of these techniques are based on a vector model of images and on the exploitation of the concepts of linear dependence and linear independence.

Furthermore, the techniques are compatible with physical photometry. A detailed description of the proposed detector and three state-of-the art change detectors is also provided. For the purposes of comparison, an evaluation procedure is presented consisting of both objective and subjective parts. This evaluation procedure results in a final performance value for each detector analyzed.
\end{abstract}

Keywords-Background extraction, change detection, illumination invariance, real-time, surveillance, Wronskian.

\section{INTRODUCTION}

Change detection offers the possibility of sensing and analyzing information about dynamic developments in a scene. These developments could be due to movements, appearance and disappearance of objects in the scene or even a total change of the scene.

The problem of change detection arises in many areas of image processing, which may be classified as follows:

- Intruder Surveillance: The aim here is to detect unusual changes and behavior in the scene for the purpose of generating an alarm. Regazzoni [1] and Foresti [2] provide examples of complete surveillance systems including change detection, object recognition, and tracking. Recently, a special section on video surveillance was published [3]. It gives an overview of video surveillance applications, but is not meant to be a study on change detection. In this paper, we

Manuscript received October 10, 2000; revised April 27, 2001.

The authors are with the Signal Processing Laboratory, Swiss Federal Institute of Technology (EPF), 1015 Lausanne, Switzerland (e-mail: emrullah.durucan@epfl.ch; Touradj.Ebrahimi@epfl.ch).

Publisher Item Identifier S 0018-9219(01)08436-5. will focus on change detection methods for intruder surveillance systems.

- Virtual Presence: Many applications require methods for combining actors with artificially generated studios and environments [4]. Through change detection, moving objects could be extracted for the purpose of superposing them in other environments.

- Compression: Narrow bandwidth communication channels limit the amount of information that can be exchanged. For real-time applications such as traffic observation from remote control rooms, this limitation is unacceptable. A way to reduce the amount of information is to detect changes and transmit only the latter or their features (e.g., moving cars, persons) [5], [6].

In Section II, objective requirements that every change detection algorithm (in all the above mentioned application areas) should meet are defined and discussed. Then, subjective requirements arising from surveillance circumstances are formulated. Based on these requirements, an evaluation procedure for surveillance-related algorithms is defined. Robustness against global variations in illumination conditions is an important requirement for change detection algorithms. Section III investigates basic photometric principles. Having these investigations in mind, we develop a photometrical way to compare two light sources illuminating a surface.

In Section IV, state-of-the art models from Skifstad [8], Hsu [9], and Aach [10] are presented. These models can be categorized as follows.

- Physical: Skifstad's adaptation of Phong's [7] illumination model is based on basic aspects of reflection of light from surfaces.

- Polynomial Modeling: Hsu models the image as a mosaic of patches where the gray-value function within each patch is described as a second order bivariate polynomial of the pixel coordinates.

- Statistical: Aach compares several test statistics and, by assuming null hypothesis, carries out a significance test. 
A vector model of images is introduced in Section V. The linear dependence and independence characteristics of the vectors is then used to define the linear dependence change detector. The Wronskian change detector, which is also based on the same linear dependence and independence concepts, is developed in Section VI. The discussion of the results and their comparison to the state-of-the art methods appear in Section VII.

\section{Evaluation ReQuirements for Change DeteCtion}

The performance assessment of video segmentation algorithms can be categorized as follows.

- Objective: Villegas [11] and Wollborn [12] focus on the design of objective figures of merit such as spatial accuracy, tracking continuity and temporal coherence. These figures of merit are difference measures calculated between a segmentation mask and a reference segmentation mask.

- Subjective: McKoen [13] proposes an application oriented evaluation method related to surveillance segmentation. Subjects were asked to rate requirements such as segmentation and tracking performance, illumination invariance, etc., for various approaches. A variance analysis method is then applied to these ratings to extract statistically significant differences between algorithms.

The above-mentioned assessment methods are designed for segmentation purposes and focus either objective or subjective figures of merit to asses the segmentation quality. Change detection, however, cannot be treated as a general segmentation algorithm; focusing on a single assessment method, objective or subjective, could lead to biased results.

Therefore, we have chosen to develop a change detection assessment method incorporating both objective and subjective requirements.

\section{A. Objective Assessment Requirements}

1) Computational Complexity: We do not incorporate any sophisticated complexity evaluation methods (such as the number of algorithmic operations, resource and memory requirements, or branching). The basic hypothesis will be that the algorithm has at its disposal all the resources provided by a given execution environment [14]. Then, for this environment and based on the above hypothesis, the computational complexity depends only on the time $t_{i}$ needed for the execution of task $i$ for a given change detection algorithm.

All change detectors have been implemented in $\mathrm{C}++$ and executed on a Sun-Ultra 1 workstation with $64 \mathrm{MB}$ of RAM and 143-MHz processor. The change detection was performed on the Y component of the YUV color space. Notice that the images were supplied in RGB format and, thus, we had to perform a color-conversion to YUV format. Thus, the execution times will be much faster when the illuminance value $\mathrm{Y}$ is directly supplied.

2) Object and Illumination Change: Skiftad [8] describes a test in which his algorithm would fail. In this test,

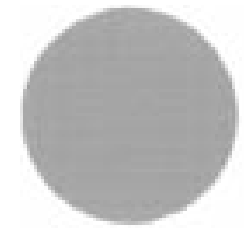

(a)

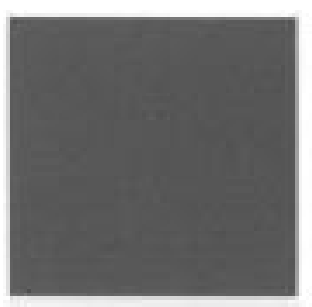

(c)

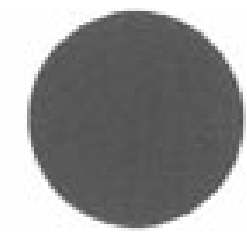

(b)

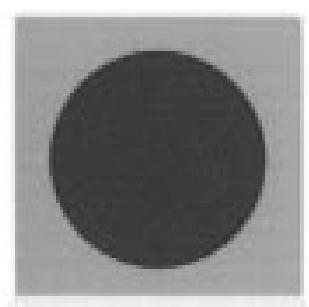

(d)
Fig. 1. (a), (b) are illustrating uniform object change and (c), (d) show an illumination change with object intrusion.

Table 1

Objective Assessment Requirements. The Importance of the Requirements Are Expressed With a Cost Weight $W$. The Cost Weight Is Used in the Evaluation

\begin{tabular}{l||l|l}
\hline$O_{i}$ & Objective Requirement & W \\
\hline \hline $\mathrm{O}_{1}$ & Computational Complexity & 5 \\
\hline $\mathrm{O}_{2}$ & Detection of object change (Fig. 1(a)) & 5 \\
\hline $\mathrm{O}_{3}$ & Detection of illumination change (Fig. 1(c)) & 5 \\
\hline
\end{tabular}

a light gray ball is replaced by a dark gray ball of the exact same size and shape under the condition of constant illumination as illustrated in Fig. 1(a). This test will be kept as a first assessment method. It actually indicates the sensitivity of the change detectors regarding change in uniform objects.

In addition to the above test, a general way to examine the response to illumination invariance is also introduced. A dark image is replaced by a light gray ball subject to the condition that the background becomes brighter [see Fig. 1(c)]. A score $G$ is then given to each algorithm depending on the degree of fulfillment of the above requirements. The score is:

- 0 when nothing or every pixel is detected;

- 1 when parts of the contour is detected;

- 2 when the contour is closed;

- 3 when object is fully detected.

A further weighting is also applied to these results depending on the relative importance of each requirement when compared to the others (objective and subjective). The value of the weight $W$ varies between one and five. In the evaluations carried out in this paper, the value of the weights for all objective requirements was set to five. This avoids any a priori bias toward any specific objective requirement (see Table 1).

\section{B. Subjective Assessment Requirements}

The algorithms were designed based on experience gained from surveillance projects at EPFL. These projects included collaboration with other universities and companies active in the field of surveillance. The companies that provided 


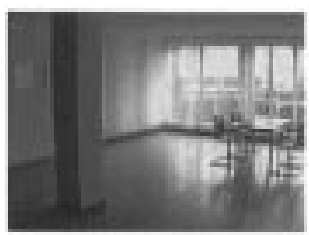

(a)

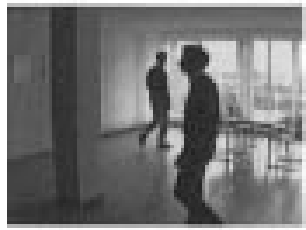

(e)

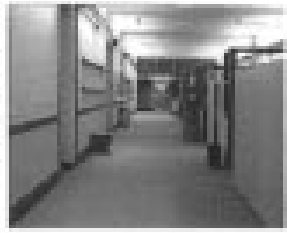

(b)

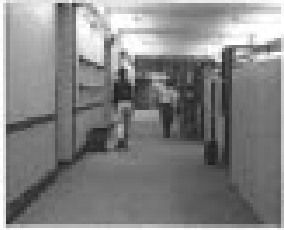

(f)

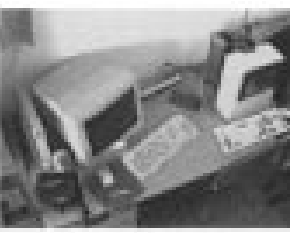

(c)

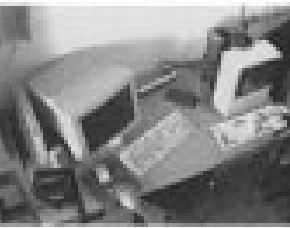

(g)

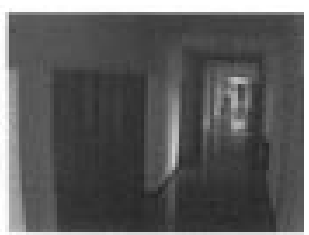

(d)

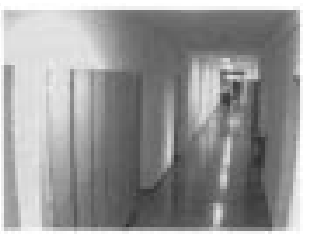

(h)

Fig. 2. Original images from the sequences Corridor, Room, Hall, and Hand.

surveillance sequences also produced a list of capabilities for the detection algorithms. In the following, these sequences will be presented and the requirements will be defined. The sequences, along with the requirements, will form our subjective assessment requirements.

1) Surveillance Sequences: In general, surveillance test sequences are real-world sequences from indoor or outdoor scenes. In this paper, although tests of outdoor situations are also provided, we will concentrate on the four indoor surveillance sequences (see Fig. 2).

- Corridor: This sequence contains a long, dark corridor in which a person enters through a door and turns the lights on, changing the illumination. The person interferes with the changing illumination conditions, making the detection very difficult. The goal is to detect the person in the presence of the illumination changes.

- Room: This sequence is based upon a room with windows and curtains in the background. The background is bright, shadows and mirroring effects can be seen on the floor, and a pillar is in the foreground. One person passes behind and another in front of the pillar. They cross each other producing shadows and reflections. The curtains move as one person passes them. The goal here is to detect the moving persons without detecting the various illumination influences such as shadows, reflections, etc. Furthermore, the algorithm under test should suppress the moving curtains-they belong in the category of background effects. Misidentification of background effect can disturb the detection and tracking of the object(s) of interest.

- Hall: This is another corridor sequence. Two persons enter the corridor. All colors are washed out. A person leaves a suitcase in the corridor and the other takes a monitor away from a table. The aim here is to detect the moving persons (with their shadows suppressed), the suitcase left in the corridor, and the missing monitor.

- Hand: This sequence consists of a close-up view of an office desk, with a hand removing a pencil from it. The goal is to detect the missing pencil.

2) Surveillance Requirements: The main focus of video surveillance systems is to develop algorithms for fast and robust detection of moving persons and objects in indoor and outdoor applications. Related change detection algorithms should provide a first-level analysis of the image sequences captured by a video surveillance camera. The reliable identification of regions belonging to an object in the acquired image sequence is the basis for further analysis in video surveillance applications. This further analysis (e.g., person identification, alarm generation, transmission, etc.) is not discussed here.

The candidate change detection algorithms should be robust against illumination changes (the switching on or off of room lights, changes in the sun's position, flickering of torches, etc.) and shadows. This is important for applications where video information is used for alarm generation; illumination changes should not generate an alarm. Furthermore, shadows or reflections of moving persons could influence tracking algorithms.

Background variations such as randomly moving curtains and plants could influence the detection results. The suppression of these kinds of changes is beyond the scope of a simple change detection algorithm; a further stage of discrimination between various types of changes is necessary. However, the response of the change detection algorithm to such changes should be considered, as it affects the work required by subsequent analysis stages.

For practical application, a change detection algorithm should also be able to cope with low-cost optics and image sensors. Therefore, robustness against noise and the ability to cope with low quality images is an important issue in many surveillance applications. Table 2 lists the subjective requirements used in this paper.

In the state-of-the art performance assessment method, McKoen [13] proposes a statistical method requiring a threshold to calculate the performance differences of algorithms with regard to requirements stated in the mentioned paper. The performance function for one sequence $p_{\nu}$ is a sum of three objective and $n$ subjective requirements (see Table 3)

$$
p_{v}=\sum_{i=1}^{3} p_{o i}+\sum_{j=1}^{n} p_{s j}
$$


Table 2

Subjective Algorithm Requirements. Their Importance in the Evaluation Is Indicated by a Cost Weight $W$

\begin{tabular}{l||l|l}
\hline$S_{j}$ & Subjective Requirement & W \\
\hline \hline S 1 & $\begin{array}{l}\text { Separates semantic objects from static back- } \\
\text { ground }\end{array}$ & 5 \\
\hline S 2 & The detection is illumination invariant & 5 \\
\hline S 3 & Associates shadows to the background. & 4 \\
\hline S 4 & Provides accurate object boundaries & 2 \\
\hline S 5 & Separates objects from moving background & 2 \\
\hline
\end{tabular}

Table 3

Table of Subjective Requirements Adapted to the Problems Present in Each of the Four Sequences. Hereby, $(x)$ Indicates That the Corresponding Requirement Is Taken Into Account and (...) Indicates That the Corresponding Requirement Is Not Considered

\begin{tabular}{c|c|c|c|c}
\hline Requirement & \multicolumn{4}{|c}{ Sequences } \\
& Corridor & Room & Hall & Hand \\
\hline \hline S1 & $\times$ & $\times$ & $\times$ & $\times$ \\
\hline S2 & $\times$ & $\ldots$ & $\ldots$ & $\ldots$ \\
\hline S3 & $\times$ & $\times$ & $\times$ & $\ldots$ \\
\hline S4 & $\times$ & $\times$ & $\times$ & $\times$ \\
\hline S5 & $\ldots$ & $\times$ & $\times$ & $\ldots$ \\
\hline \hline n & 4 & 4 & 4 & 2 \\
\hline
\end{tabular}

This function does not require a threshold since it sums the performance for the various objective $p_{o}$ and subjective $p_{s}$ requirements

$$
p_{o i, s j}=W_{i, j} G_{i, j}
$$

where

$$
\begin{aligned}
& i, j \quad \text { objective and subjective indices from Tables } 1 \text { and } \\
& 2 \text {, respectively; } \\
& W_{i, j} \quad \text { cost weight (see Tables } 1 \text { and 2); } \\
& G_{i, j} \text { score obtained reflecting the degree of fulfillment }
\end{aligned}
$$

The performance function (1) is a sum over the three objective and up to five subjective requirements. Equation (1) provides a performance measure for only one sequence. In case of several sequences, the following formula could provide a performance measure:

$$
P=\sum_{v=1}^{N_{v}} p_{v}
$$

where

$N_{\nu} \quad$ number of sequences tested;

$p_{\nu} \quad$ sequence performance from (1);

$\nu \quad$ index for the sequence.

In this paper, four sequences were used for evaluations. The overall performance will be calculated as a percentage of the maximum possible $P$ value. In our case, $P=251$.
Table 4

Description of the Grades and the Cost Weights

\begin{tabular}{l||l||l|l}
\hline$G$ & Grade $G$ & $W$ & Cost Weight $W$ \\
\hline \hline 4 & Excellent & 5 & Prime importance \\
\hline 3 & Meets requirement & 4 & Important \\
\hline 2 & Is acceptable & 3 & Good to have \\
\hline 1 & Almost acceptable & 2 & Low importance \\
\hline 0 & Fails completely & 1 & Not important \\
\hline
\end{tabular}

Table 5

Photometric and Radiometric Terms

\begin{tabular}{ll}
\hline Energy related & Photometry related \\
\hline \hline Radiant energy $Q$ & Luminous energy \\
\hline Radiant flux $\Phi$ & Luminous flux \\
\hline Irradiance $E$ & Illuminance \\
\hline Radiant Intensity $I$ & Luminous intensity \\
\hline
\end{tabular}

\section{The Physics OF ILLUMINATION}

Before starting to develop change detection algorithms it is worth understanding what kind of physical information is present in images and what can be measured with this information. In classical image processing, the values of the pixels correspond to the energy deposed by the light received by the imaging system. Physical photometry is the science concerned with the measurement of light and its impact on an imaging system, e.g., human visual system [15]. Photometry also enables the comparison of two light sources that could be exploited for change detecting purposes.

\section{A. Principles of Photometry}

We start this section by providing some basic definitions of photometry.

Luminous Flux: The total energy that emerges per unit time from a point-like source is expressed by $\Phi$ (see Table 5 from Jaehne [15]) expressed as $\Phi=d Q / d t$.

Luminous Intensity: The luminous intensity $I$ of a source is the luminous flux radiated per solid angle $\Omega$, $I=d \Phi / d \Omega$. The solid angle is measured in steradians $(s r)$ [see Fig. 3(a)]. The size of the solid angle is given by the quotient

$$
d \Omega=\frac{d S^{\prime} \cos \theta^{\prime}}{r^{2}}
$$

where $\theta^{\prime}$ represents the angle that is made between the surface normal of $d S^{\prime}$ and $r$.

Illuminance: The illuminance is the luminous flux deposed per unit area over a surface element $d S^{\prime}$. In the case of a point-like source $P$ [see Fig. 3(b)], i.e., $r$ and $\Theta^{\prime}$ do not change significantly, then

$$
E^{\prime}=\frac{d \Phi}{d S^{\prime}}=I \frac{\cos \Theta^{\prime}}{r^{2}} .
$$

Equation (5) is the basic equation of practical photometry. It expresses the cosine law of illumination (E proportional to 


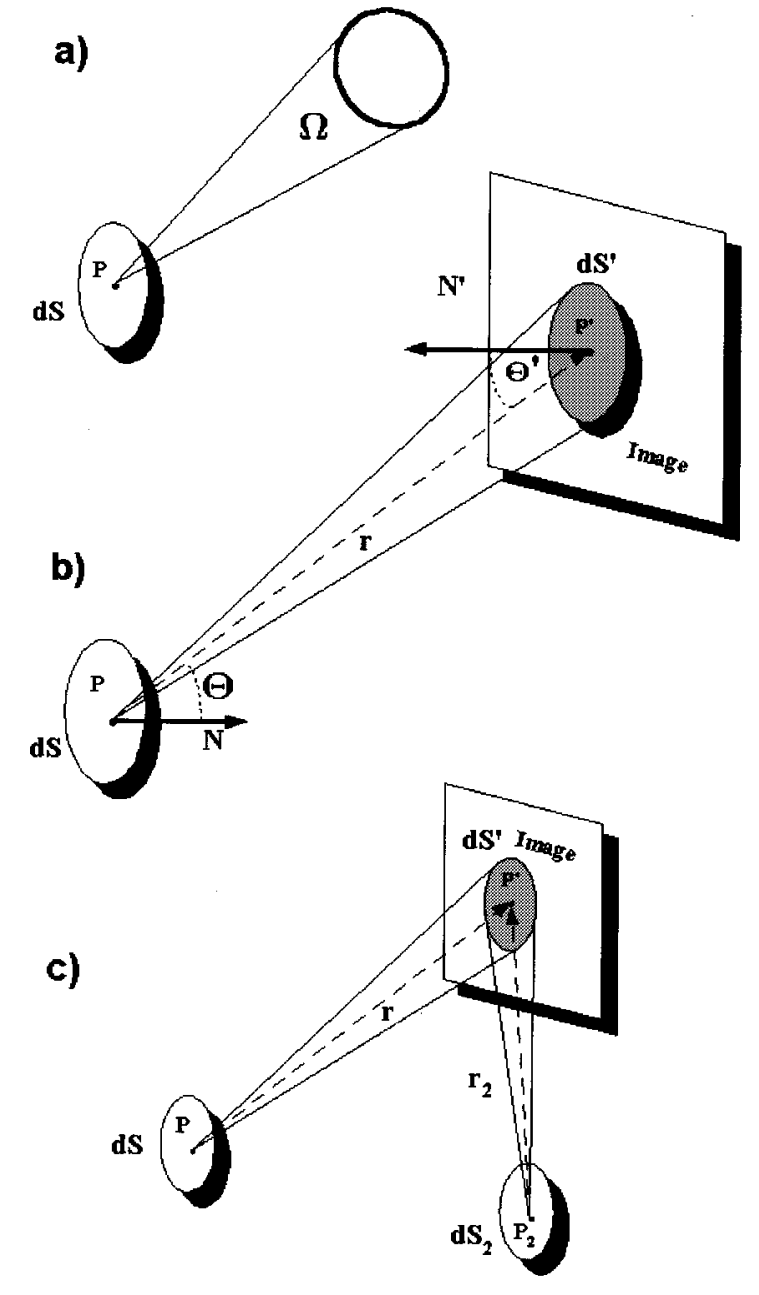

Fig. 3. The apex of the solid angle $\Omega$ is located at point $P$ (a). Illumination of the surface $d S^{\prime}$ by a point located in $d S$ (b). A second source $d S_{2}$ is illuminated by the same surface (c).

$\cos \Theta^{\prime}$ ) and the inverse square law (E inversely proportional to $r^{2}$ ). This equation enables the comparison of luminous intensities originating from two light sources [16].

Notice that these formulas are defined for monochromatic light. For nonmonochromatic light sources, a weighted integral must be performed over the entire visible light spectrum. Nevertheless, the ideas developed here remain the same.

\section{B. Comparison of Luminous Intensities in Physics}

For the purpose of determining an unknown luminous intensity $I_{1}$ from a given source (e.g., a star) a reference light source with known intensity $I_{2}$ is applied. Both light sources illuminate a surface with illuminances $E_{1}^{\prime}$ and $E_{2}^{\prime}$, respectively. The illuminance of the reference light source $E_{2}^{\prime}$ is then adjusted such that it equals the illuminance $E_{1}^{\prime}$

$$
\frac{I_{1} \cos \Theta_{1}^{\prime}}{r_{1}^{2}}=\frac{I_{2} \cos \Theta_{2}^{\prime}}{r_{2}^{2}} .
$$

Since the distances $r_{1}, r_{2}$ and the angles $\Theta_{1}^{\prime}, \Theta_{2}^{\prime}$ are known, the intensity $I_{1}$ can easily be calculated. A photometer is an instrument where such experiments are performed. A simple photometer was designed by Lummer and Brodhun [17].

\section{Comparison of Illuminances in Change Detection}

If we wish to track changes in equal area regions from two images, we can compare the luminous intensities of these regions by calculating the ratio of the illuminances

$$
\frac{E_{1}^{\prime}}{E_{2}^{\prime}}=\frac{\frac{I_{1} \cos \Theta_{1}^{\prime}}{r_{1}^{2}}}{\frac{I_{2} \cos \Theta_{2}^{\prime}}{r_{2}^{2}}} .
$$

If there has been no change, then (7) reduces to ${ }^{1}$

$$
\frac{E_{1}^{\prime}}{E_{2}^{\prime}}=1 \text {. }
$$

Fig. 3 illustrates a surface illuminated by two sources.

In general, (8) is valid for all surface elements (in the area of interest) $d S_{i}^{\prime}$ leading to the relation

$$
\frac{E_{1, i}^{\prime}}{E_{2, i}^{\prime}}=1
$$

where $E_{1, i}^{\prime}$ denotes the illuminance deposed in surface element $i$ in the first image and $E_{2, i}^{\prime}$ denotes the illuminance deposed in surface element $i$ in the second image. Thus, (9) is a physical ratio that enables the measurement of changes between images.

\section{State-OF-the Art Change Detection Methods}

The most intuitive technique to detect change is simple differencing followed by thresholding [19], [20]. Change in a pixel is detected if the difference in gray levels of the corresponding pixels in consecutive images exceeds a preset threshold. Since only one pixel is considered at a time, this technique has the advantage of low computational cost. Nevertheless, the result is very susceptible to noise. Hence, it is not suitable for applications where high precision is required. In order to circumvent this problem and achieve more robust change detection, different approaches have been proposed [8], [9], [21]-[23]. Among them, some characteristic functions are defined and computed for multipixel regions to detect changes. Such schemes may be referred to as regionbased schemes. The advantage of a region-based scheme is that the adverse effect of noise in detecting changes can be reduced.

Examples of such region based methods are the Shading Model, the Statistical Change Detection method, and the Derivative Model proposed by Skifstad and Jain [8], Aach et al. [10], and Hsu et al. [9], respectively. These three state-of the art methods will be tested and will serve as a benchmark for the proposed change detection algorithms.

\section{A. Shading Model (SM)}

The shading model (SM) is based on the technique proposed by Skifstad. He uses the fact that the intensity at a given point on an object $I_{p}^{2}$ is the product of the illumination $I_{i}$ and

\footnotetext{
${ }^{1}$ Here, we neglect physical effects such as atmospheric attenuation, multiple scattering, light interfering from other areas, etc.

${ }^{2}$ In all of the state-of-the art change detection methods, the original terminology of the respective authors is used.
} 
a shading coefficient $S_{p}$. Thus, the intensity is calculated for each point

$$
I_{p}=I_{i} S_{p}
$$

This model was first introduced by Oppenheim et al. [26] and is commonly used in computer graphics.

The main assumption of Skiftad is that when there is no physical change between two given areas of two images, then the intensity ratios of (10) becomes

$$
\frac{I_{p 1}}{I_{p 2}}=\frac{I_{i 1}}{I_{i 2}}
$$

which does not depend on the shading coefficients. The terms $I_{p 1,2}$ and $I_{i 1,2}$ denote the intensities and illuminations on a object point in the first and the second image, respectively. In contrast, if there is a physical change between these two areas then (11) is not valid.

For the detection of changes with the SM, a reference and a test image are cut in fixed blocks of areas of interest (AOIs). The variance of the intensity ratios for each recorded intensity in the corresponding AOIs of the two images are calculated. If the value of the variance exceeds a predetermined threshold, the test image is marked as changed. Typical thresholds of the SM are given in Table 11.

1) Results for Shading Model: Fig. 4 illustrates the change detection masks obtained by SM. The name and the number of the masks corresponds to the name of the sequence and the frame number of the current image in Fig. 2. It can be seen that the SM has difficulties in detecting semantic objects and their interiors and the object boundaries are very discrete and do not necessarily correspond to those of real object. But the algorithm is illumination invariant and removes shadows and reflections successfully.

The SM is a computationally complex algorithm. Its execution time is given in Table 12. Table 6 lists the scores obtained by this algorithm for each given assessment requirement.

The sequence performance values $p_{i}$ and the overall performance value $P$ are calculated in Table 13 .

\section{B. The Derivative Model (DM)}

The change detector of Hsu et al. [9] models images as a mosaic of regions where the gray-value function within each region is described as a second-order bivariate polynomial of the pixel coordinates. Skiftad and Jain use partial derivatives on these second-order surface models to render Hsu's model illumination invariant. The change detection difference metric $D$ for each region $A$ is defined by

$$
D=\sum_{x, y \in A} f_{1}(x, y)-f_{2}(x, y)
$$

where the index indicates the relevant image and the metric $f$ defined as

$$
f_{i}=\frac{\partial g_{i}(x, y)}{\partial x}+\frac{\partial g_{i}(x, y)}{\partial y} .
$$

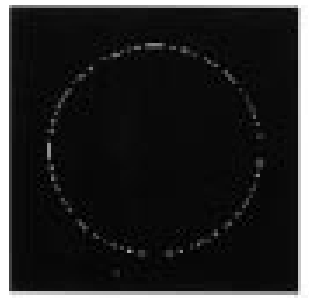

(a)

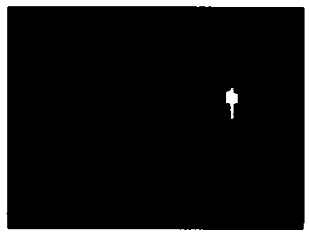

(c)

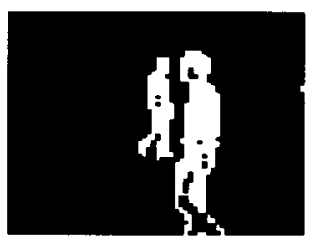

(e)

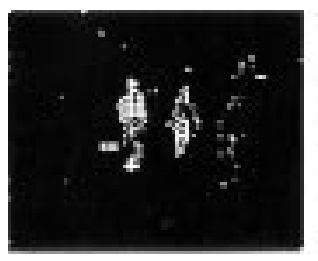

(g)

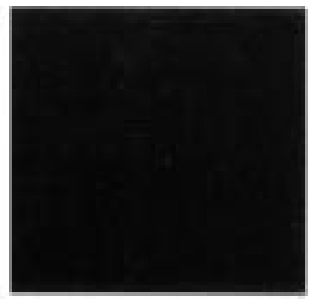

(b)

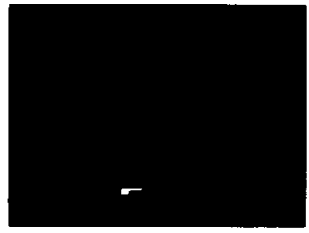

(d)

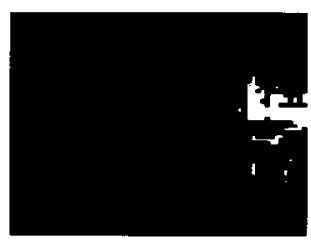

(f)

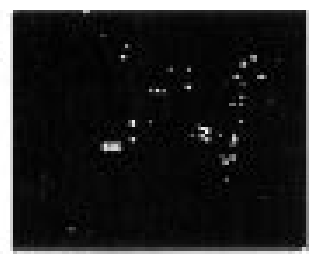

(h)
Fig. 4 Change detection results of the SM.

Table 6

Subjective and Objective Scores Obtained by SM

\begin{tabular}{c|c|c|c|c}
\hline Requirement & \multicolumn{5}{|c}{ Sequences } \\
& Corridor & Room & Hall & Hand \\
\hline \hline S1 & 3 & 2 & 2 & 3 \\
\hline S2 & 3 & $\ldots$ & $\ldots$ & $\ldots$ \\
\hline S3 & 3 & 3 & 3 & $\ldots$ \\
\hline S4 & 1 & 1 & 1 & 2 \\
\hline S5 & $\ldots$ & 2 & 1 & $\ldots$ \\
\hline \hline 01 & \multicolumn{5}{|c}{1} \\
\hline 02 & \multicolumn{5}{|c}{1} \\
\hline 03 & & \multicolumn{5}{|c}{} \\
\hline \hline
\end{tabular}

The gray-value surface function $g_{i}(x, y)$ for the $i$ th frame is defined as

$$
g_{i}(x, y)=a_{00}+a_{10} x+a_{01} y+a_{11} x y+a_{20} x^{2}+a_{02} y^{2} .
$$

Since there are no implementations proposed for the DM by Skifstad and Jain, we implemented this model as follows: A reference and a current frame are partitioned in fixed blocks of equal size. For each block, there are six unknown coefficients $\left(a_{00}, a_{10}, a_{01}, a_{11}, a_{20}, a_{02}\right)$ that have to be determined. Thus, six functions are necessary to determine them. We calculated the functions $g_{i}$ on $3 \times 2$ areas. With these six gray-values taken by the functions, the determination of the 
coefficients is straightforward. Then, these coefficients are resubstituted into the surface function 14 .

The derivative according to (13) is taken and the value of $f_{i}(x, y)$, with $x, y \in A$, is defined for each block $A$. The procedure to define the functions $f_{i}(x, y)$ for the reference frame $i=1$ and the current frame $i=2$ are done separately. The $f_{1}(x, y)$ are calculated once for the whole sequence and only the $f_{2}(x, y)$ have to be calculated for each current frame. This reduces the computational complexity considerably. When the difference of $f_{1}(x, y)$ and $f_{2}(x, y)$ [see (12)] in a particular block are greater than a given threshold, then a change occurred in that given block. Typical thresholds and the block size of the DM are given in Table 11.

1) Results for Derivative Model: Fig. 5 illustrates the change detection masks obtained by the Derivative Model. It has fundamental difficulties in detecting semantic objects and their interiors and detected boundaries are very discrete and do not necessarily correspond to that of real objects. The algorithm is however illumination invariant and removes shadows and reflections. The DM is not a computationally complex method. It's execution times are given in Table 12.

Table 7 lists the scores obtained by this algorithm for each given assessment requirement.

The sequence performance values $p_{i}$ and the overall performance value $P$ are calculated in Table 13.

\section{Statistical Change Detection (SCD)}

The main idea behind this approach is to find a statistical description of an ensemble of pixels that enables the detection of changes. It assumes that the change detection could be treated as a statistical hypothesis testing with:

- Hypothesis $H_{0}$ : no change occurred at pixel $x$.

- Hypothesis $H_{1}$ : a change occurred at pixel $x$.

It further assumes that the image noise can be represented by either a Gaussian or a Laplacian distribution. Following this assumption, a noise probability density function is calculated. In this paper, only Laplacian noise is taken into account since it provided better change detection results. This observation is consistent with the results obtained by Toth $e t$ al. [27].

If no change has occurred between the images, then the white noise in the gray-level difference $d_{k}$ at spatial locations $k$ is given by

$$
d_{k}=y_{1}(k)-y_{2}(k)
$$

where $Y_{1,2}=\left\{y_{1,2}(k)\right\}$ are the two considered images and $d_{k}$ is a Laplacian distribution. The hypothesis testing is done by

$$
\Delta_{i}=\frac{\sqrt{2}}{\sigma_{0}} \sum_{k \in w_{i}}\left|d_{k}\right|
$$

where $\Delta_{i}$ is the sum of absolute differences within a sliding window $w_{i}$, of size $N$ pixels and centered at position $i$. De-

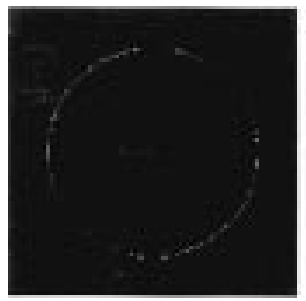

(a)

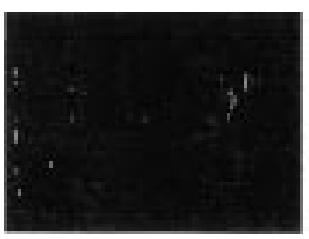

(c)

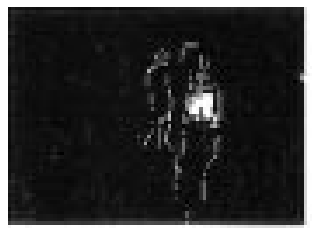

(e)

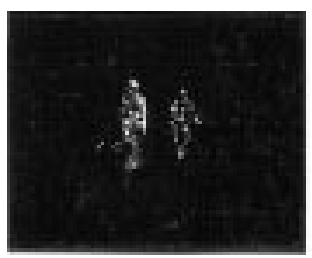

(g)

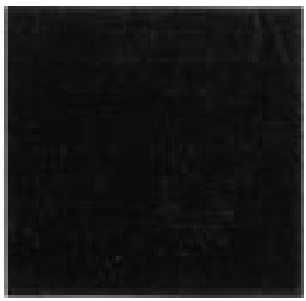

(b)

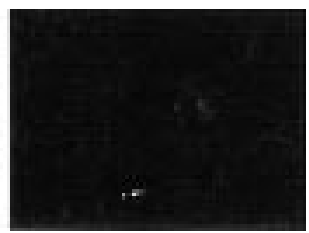

(d)

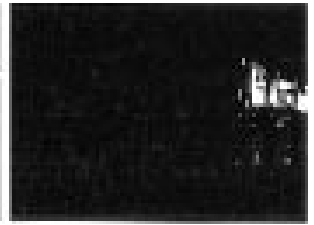

(f)

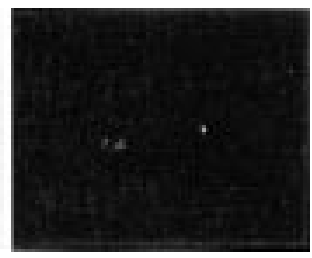

(h)
Fig. 5. Change detection results of the DM.

Table 7

Subjective and Objective Scores Obtained by DM

\begin{tabular}{c|c|c|c|c}
\hline Requirement & \multicolumn{5}{|c}{ Sequences } \\
& Corridor & Room & Hall & Hand \\
\hline \hline S1 & 3 & 1 & 2 & 2 \\
\hline S2 & 3 & $\ldots$ & $\ldots$ & $\ldots$ \\
\hline S3 & 3 & 3 & 3 & $\ldots$ \\
\hline S4 & 1 & 1 & 2 & 1 \\
\hline S5 & $\ldots$ & 2 & 1 & $\ldots$ \\
\hline \hline 01 & \multicolumn{5}{|c}{5} \\
\hline 02 & \multicolumn{5}{|c}{0} \\
\hline 03 & \multicolumn{5}{|c}{} \\
\hline
\end{tabular}

pending on the value of $\Delta_{i}$ a decision about the change is made, i.e.,

$$
\begin{aligned}
& \Delta_{i}<T: 0 \text { unchanged at } \mathrm{k} \\
& \Delta_{i}>T: 1 \text { changed at } \mathrm{k}
\end{aligned}
$$

where $T$ denotes a threshold value.

The noise standard deviation $\sigma_{0}$ of the gray-level differences $d_{k}$ is assumed to be constant over space. Given the hypothesis $H_{0}$, the threshold can be determined from the following significance test

$$
\alpha=\operatorname{Prob}\left(\Delta_{i}>T \mid H_{0}\right) .
$$




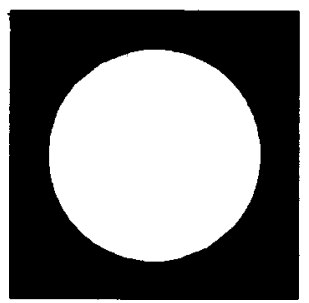

(a)

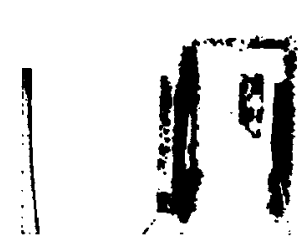

(c)

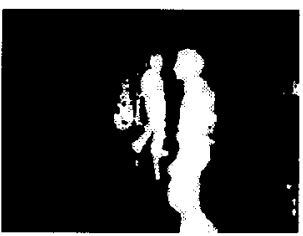

(e)

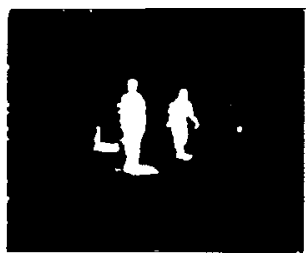

(g) (b)

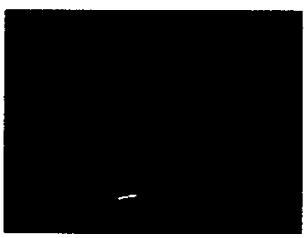

(d)

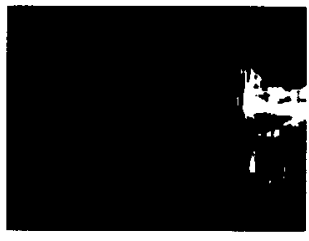

(f)

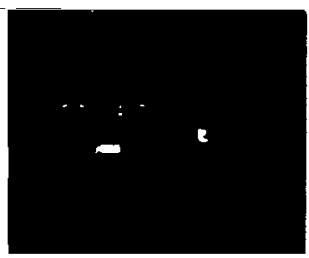

(h)
Fig. 6. Change detection results of the SCD.

The significance value $\alpha$ is equivalent to the probability that $\Delta_{i}$ exceeds the threshold $T$, given the hypothesis $H_{0}$ that $d_{k}$ is due to noise only and not a significant change. For a fixed false alarm rate $\alpha$, the threshold $T$ can be obtained from tables of the $\chi^{2}$ distribution [10], [27].

Typical values for the significance level $\alpha$ are given in Table 11, image noise $\sigma_{0}$ varies around ten.

1) Results for Statistical Change Detection: Fig. 6 illustrates the change detection masks obtained by this method. It detects semantic objects and their interiors. The object boundaries are closed. Unfortunately it has significant difficulties with changing illumination conditions in general and with shadows and reflections in particular.

The SCD is of moderate computational complexity. Its execution time is given in Table 12. Table 8 lists the scores obtained by this algorithm for each given assessment requirement.

The sequence performance values $p_{i}$ and the overall performance value $P$ are calculated in Table 13 .

\section{LINEAR DEPENDENCE AND VECTOR MODEL (LDD)}

The proposed vector model of images is an empirical method. The advantage of the vector model is that it incorporates the concept of linear (in)dependence.
Table 8

Subjective and Objective Scores Obtained by the SCD

\begin{tabular}{c|c|c|c|c}
\hline Requirement & \multicolumn{5}{|c}{ Sequences } \\
& Corridor & Room & Hall & Hand \\
\hline \hline S1 & 0 & 1 & 3 & 4 \\
\hline S2 & 0 & $\ldots$ & $\ldots$ & $\ldots$ \\
\hline S3 & 0 & 0 & 0 & $\ldots$ \\
\hline S4 & 0 & 1 & 3 & 4 \\
\hline S5 & $\ldots$ & 1 & 2 & $\ldots$ \\
\hline \hline 01 & \multicolumn{5}{|c}{2} \\
\hline 02 & \multicolumn{5}{|c}{0} \\
\hline 03 & \multicolumn{5}{|c}{0} \\
\hline \hline
\end{tabular}
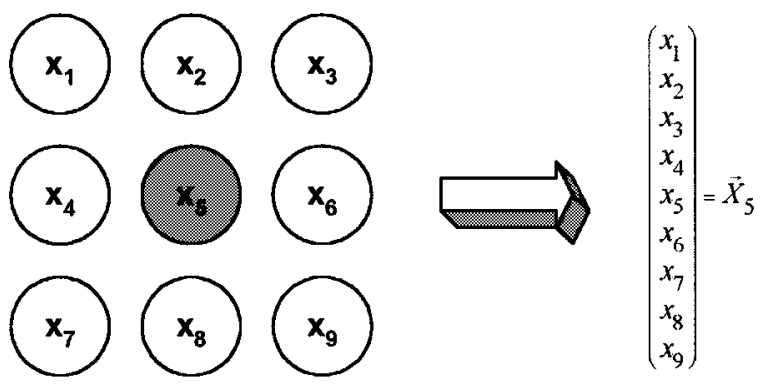

Pixel

Vector

Fig. 7. Illustration of how the vectors are constructed. The region of support for the vector is $3 \times 3$.

\section{A. The Vector Model of Images}

The vector model is illustrated in Fig. 7. Each pixel with its neighboring area is the region of support for the corresponding vectors. The center pixel of a region of support is then replaced by the vectors. The region of support can have various sizes, e.g., (a) $3 \times 3$, (b) $5 \times 5$, and (c) $7 \times 7$.

The components of vectors correspond to the illuminance values stored in each pixel of the image, i.e., $x_{5}=E_{1,5}^{\prime}$.

By modeling the image as an ensemble of vectors changes in their lengths or directions (with respect to the initial unchanged status) can be exploited. Mathematically, this corresponds to the concepts of linear dependence and linear independence respectively. The decision as to whether a vector is linearly dependent on another provides the possibility to decide whether there has been a change or not.

\section{B. The Linear Dependence Change Detector}

Definition 5.1: Let $\mathcal{V}$ be a $\mathbb{K}$-vector space. A finite set $\left\{\vec{X}_{1}, \ldots, \vec{X}_{n}\right\}=\left\{\vec{X}_{i}\right\}_{i=1}^{n}$ of a vector space $\mathcal{V}$ with coefficients $k_{i} \in \mathbb{K}$ is linearly independent if and only if

$$
\sum_{i=1}^{n} k_{i} \vec{X}_{i}=0, \quad \text { implies that } \quad\left\{k_{i}=0\right\}_{i=1}^{n}
$$

otherwise it is said to be linearly dependent [28].

In the case of two vectors $\vec{X}$ and $\vec{Y} \in \mathcal{V}$, originating from a reference and a current image respectively, Definition 5.1 is still valid, i.e., $\overrightarrow{0}=\vec{X}-k \cdot \vec{Y}$. For these two vectors, Remark 5.1 follows immediately [29]. 
Remark 5.1: Let $\vec{X}=\left(x_{1}, \ldots, x_{n}\right), \quad \vec{Y}=$ $\left(y_{1}, \ldots, y_{n}\right) \in \mathcal{V}$ be two linearly dependent vectors with no components zero, then the ratio of their components is constant, i.e., $x_{1} / y_{1}=\cdots=x_{n} / y_{n}=k$.

It follows that the variance $\sigma^{2}$ below must be zero

$$
\sigma^{2}=\frac{1}{n-1} \sum_{i=1}^{n}\left(\frac{x_{i}}{y_{i}}-\mu\right)^{2} \text { with } \mu=\frac{1}{n} \sum_{i=1}^{n} \frac{x_{i}}{y_{i}} .
$$

Following the ideas above, a criterion for change between a reference and a current image can be introduced for the Linear Dependence Detector (LDD):

- $\sigma^{2}=0 ; \vec{X}$ and $\vec{Y}$ are linearly dependent, i.e., no change has occurred;

- $\sigma^{2}>0 ; \vec{X}$ and $\vec{Y}$ are linearly independent, i.e., no change has occurred.

\section{Photometry and Linear Dependence of Vectors}

Remark 5.1 states that in the case of two linearly dependent vectors, all their component ratios are equal and constant. This is similar to the result described in Photometry (see Section III-A) for two light sources illuminating an area on the surface with $n$ points

$$
k=\frac{E_{1,1}^{\prime}}{E_{2,1}^{\prime}}=\cdots=\frac{E_{1, n}^{\prime}}{E_{2, n}^{\prime}}
$$

the ratio of the illuminance values is constant if no change has occurred in the scene.

For unchanged regions, the illuminance ratios remain the same and the underlying vectors are linearly dependent. This could be exploited for the extraction of the unchanged background, for example.

\section{Results for Linear Dependence Change Detector}

Fig. 8 illustrates the change detection masks produced by the Linear Dependence Change Detector. The LDD detects semantic objects but has some problems with object interiors. The object boundaries correspond to those of real objects. It is illumination invariant and removes shadows and reflections.

The LDD is of low computational complexity. The execution time is given in Table 12. Table 9 lists the scores obtained by this algorithm for each given assessment requirement.

The sequence performance values $p_{i}$ and the overall performance value $P$ are calculated in Table 13.

\section{E. Problems of the $L D D$}

The results in Section V-D reveal the problems of the LDD with the detection of object interiors. The reason for this failure lies in the variance $\sigma^{2}$ itself. The variance is zero for all constant arguments. This problem occurs for equal illuminance changes, e.g., when a uniform object enters in a uniform scene then, except for the contours, the ratio of the corresponding pixels is constant. This problem results in undetected areas in originally changed regions, as can be seen in Fig. 8 Room.

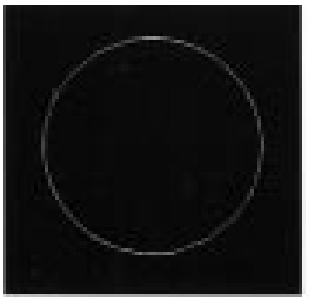

(a)

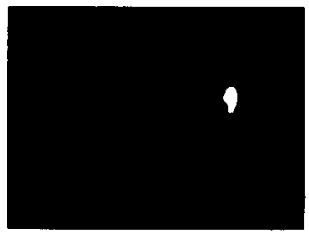

(c)

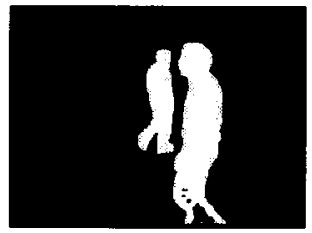

(e)

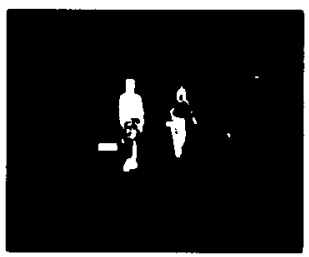

(g)

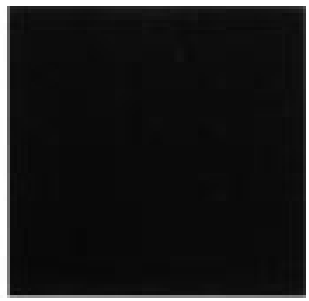

(b)

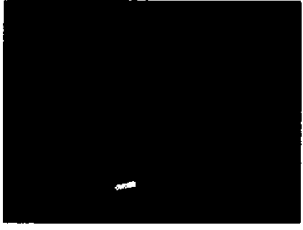

(d)

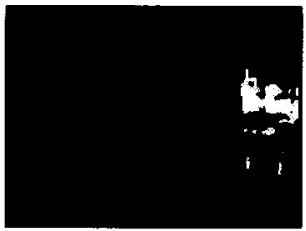

(f)

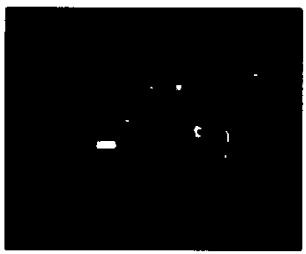

(h)
Fig. 8. Change detection results of the LDD.

Table 9

Subjective and Objective Scores Obtained by LDD

\begin{tabular}{c|c|c|c|c}
\hline Requirement & \multicolumn{5}{|c}{ Sequences } \\
& Corridor & Room & Hall & Hand \\
\hline \hline S1 & 3 & 3 & 3 & 4 \\
\hline S2 & 3 & $\ldots$ & $\ldots$ & $\ldots$ \\
\hline S3 & 3 & 3 & 3 & $\ldots$ \\
\hline S4 & 2 & 3 & 2 & 3 \\
\hline S5 & $\ldots$ & 2 & 2 & $\ldots$ \\
\hline \hline 01 & \multicolumn{5}{|c}{3} \\
\hline 02 & \multicolumn{5}{|c}{0} \\
\hline 03 & \multicolumn{5}{|c}{} \\
\hline
\end{tabular}

\section{WRONSKIAN Change Detection Model (WM)}

A simple and more rigorous test for determining the linear dependence or independence of vectors is the Wronskian determinant. The Wronskian determinant is defined for functions. Nevertheless, it can be applied on vectors [30].

Definition 6.1: A set of functions $\left\{f_{i}(x)\right\}_{i=1}^{n}$ each defined on a common interval $I$, is said to be linearly dependent on $I$, if there exist a set of constants $\left\{k_{i}\right\}_{i=1}^{n}$ such that

$$
\sum_{i=1}^{n} k_{i} f_{i}(x)=0
$$


for every $x$ on $I$. If it is not possible to find such constants, then $\left\{f_{i}(x)\right\}_{i=1}^{n}$ is linearly independent.

Definition 6.2: The Wronskian $W\left(\left\{f_{i}\right\}_{i=1}^{n} ; x\right)$ of a set of functions $\left\{f_{i}(x)\right\}_{i=1}^{n}$, each of which possesses derivatives of order $n-1$, is defined to be the following determinant:

$$
W\left(\left\{f_{i}\right\}_{i=1}^{n} ; x\right):=\left|\begin{array}{cccc}
f_{1}(x) & f_{2}(x) & \cdots & f_{n}(x) \\
f_{1}^{\prime}(x) & f_{2}^{\prime}(x) & \cdots & f_{n}^{\prime}(x) \\
\cdots & \cdots & \cdots & \cdots \\
f_{1}^{n-1}(x) & f_{2}^{n-1}(x) & \cdots & f_{n}^{n-1}(x)
\end{array}\right| .
$$

The following definition illustrates the link between the Wronskian and the concept of linear dependence.

Definition 6.3: A function $f(x)$ is said to vanish identically on an interval $I: a \leq x \leq b$, or to be identically zero on $I$, if for every $x \in I, f(x)=0$.

Theorem 6.1: If a set of functions $\left\{f_{i}(x)\right\}_{i=1}^{n}$, each of which possesses a derivative of order $n-1$, is linearly dependent on an interval $I: a \leq x \leq b$, then its Wronskian vanishes identically on I (for the proof, see [31]).

\section{A. Examples for Wronskian}

Given two components $x(E)$ and $y(E)$ of the vectors $\vec{X}$ and $\vec{Y}$. Since they are functions of the illuminance $E$ as mentioned in Section $\mathrm{V}-\mathrm{C}$, the derivative is simply $x^{\prime}=d x / d E$.

Example 6.1: The linear combination of these functions is

$$
x k_{1}+y k_{2}=0
$$

The Wronskian for this equation is then

$$
W=\left|\begin{array}{cc}
x & y \\
x^{\prime} & y^{\prime}
\end{array}\right|=x y^{\prime}-x^{\prime} y
$$

Example 6.2: Solving (21) for $k_{2}$ yields

$$
\frac{x}{y} \cdot k_{1}+1 \cdot k_{2}=0
$$

and the Wronskian is

$$
W=\left|\begin{array}{cc}
\frac{x}{y} & 1 \\
\left(\frac{x}{y}\right)^{\prime} & 0
\end{array}\right|=-\left(\frac{x}{y}\right)^{\prime}=-\frac{x^{\prime}}{y}-x\left(\frac{1}{y}\right)^{\prime}
$$

where $y \neq 0$.

\section{B. The Wronskian Change Detector}

If we further exploit the fact that the ratio of illuminance values originating from two light sources helps to quantify the differences between the light sources, then a change detection algorithm can be formulated based on Example 6.2. To detect the changes between two images, we initially assume that there has been no change, i.e., that the functions are equal $x(E)=y(E)=E$. The Wronskian of them is easily calculated

$$
0=-\frac{x^{\prime}}{y}-x\left(\frac{1}{y}\right)^{\prime}=\frac{x}{y^{2}}-\frac{1}{y}
$$

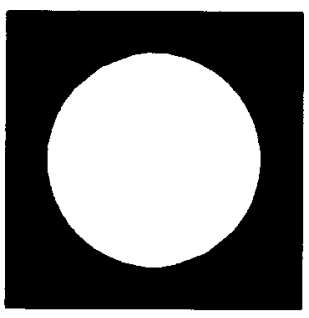

(a)

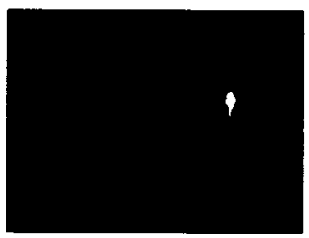

(c)

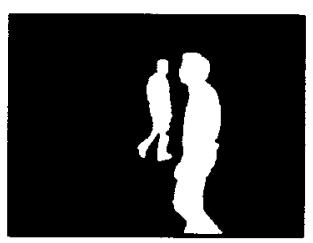

(e)

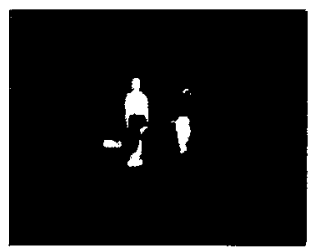

(g)

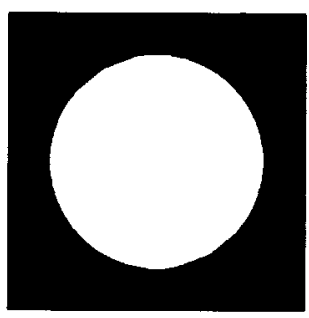

(b)

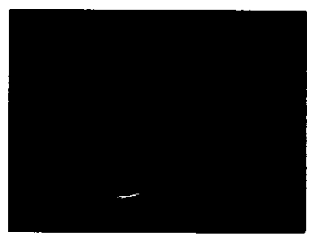

(d)

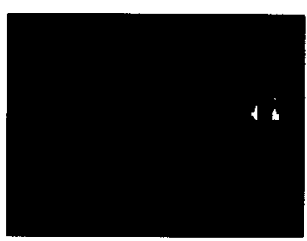

(f)

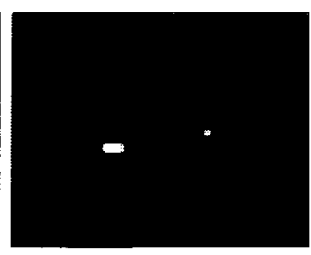

(h)
Fig. 9. Change detection results of the WM.

$$
=\frac{x^{2}}{y^{2}}-\frac{x}{y}
$$

Applying it to all components of $\vec{X}$ and $\vec{Y}$ yields

$$
W\left(\frac{x_{i}}{y_{i}}\right)=\frac{1}{n} \sum_{i=1}^{n} \frac{x_{i}^{2}}{y_{i}^{2}}-\frac{1}{n} \sum_{i=1}^{n} \frac{x_{i}}{y_{i}}=0 .
$$

The factor $1 / n$ is added to normalize the results to the vector dimensions so that same thresholds can be applied for different vector dimensions.

Equation (26) is the proposed Wronskian Model (WM) for detecting changes.

\section{Results for Wronskian}

Fig. 9 illustrates the change detection masks produced by the WM. The WM detects semantic objects and their interiors. The object boundaries are closed and correspond to those of real objects. It is illumination invariant and removes shadows and reflections.

The WM is of low computational complexity. Its execution time is shown is illustrated in Table 12. Table 10 lists the scores obtained by this algorithm for each given assessment requirement. 
Table 10

Subjective and Objective Scores Obtained by WM

\begin{tabular}{c|c|c|c|c}
\hline \multirow{2}{*}{ Requirement } & \multicolumn{5}{|c}{ Sequences } \\
& Corridor & Room & Hall & Hand \\
\hline \hline S1 & 4 & 3 & 3 & 4 \\
\hline S2 & 3 & $\ldots$ & $\ldots$ & $\ldots$ \\
\hline S3 & 3 & 3 & 3 & $\ldots$ \\
\hline S4 & 3 & 2 & 2 & 4 \\
\hline S5 & $\ldots$ & 2 & 2 & $\ldots$ \\
\hline 01 & \multicolumn{5}{|c}{4} \\
\hline 02 & \multicolumn{5}{|c}{3} \\
\hline 03 & & 3 & \\
\hline
\end{tabular}

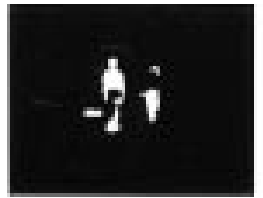

(a)

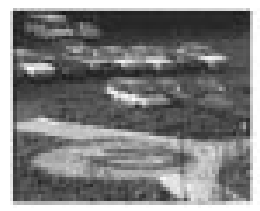

(a)

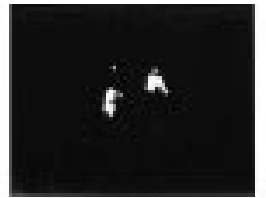

(b)

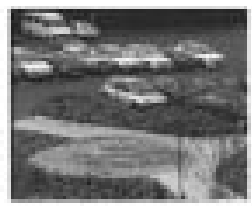

(b)

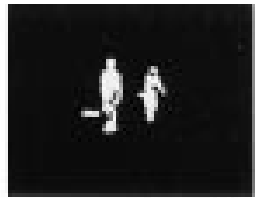

(c)

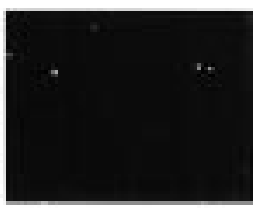

(c)
Fig. 10. Results for the Wronskian $W$ (a), the Wronskian $W^{*}$ (b), and the sum of them (c). $W^{*}$ detects only the bright changes. (d) and (e) are representing an outside illumination change (cloud covering sun). The darkening of the scene and the disappearance of the bench shadow is not detected.

The sequence performance values $p_{i}$ and the overall performance value $P$ are calculated in Table 13 .

\section{Extension of the WM}

The Wronskian $W$ is invariant against high illuminance values whether they are due to global illumination change or intruders [see Fig. 10(a)]. The separation between global illumination change and high illuminance intruders can be done by a two-step method in which the first step decides whether there has been a global illumination change and the second step calculates the Wronskian.

The test for global illumination change can be performed with the pixel illuminance ratio $k_{i}=x_{i} / y_{i}$ for the reference $x_{i} \in I_{r}$ and current image $y_{i} \in I_{c}$. The illuminance ratio is smaller than the one for high-illuminance value changes, as can be seen in Fig. 11(b). By comparing the number $n_{k}$ of $k_{i}<1$ to a global illumination change threshold $n_{g i}$, the global illumination test is performed. We assume that a global illumination change (such as turning lights on in a room) occurred when approximately $\left(n_{g i}=\right) 1 / 5$ of the total number pixels have changed. The test for such a change could also be performed with the Wronskian itself, but since it is computationally more complex than just simply calculating the ratio, the Wronskian is not suitable to do this test.

If a global illumination change occurred in the current image, then the Wronskian $W(x / y)$ of WM is calculated. The Wronskian $W(x / y)$ suppresses high illuminance values since in this case the ratio $k$ is small and, thus, is illumination invariant. If there has been no global illumination change, then the Wronskian $W(x / y)$ and its inverse ratio $W^{*}(y / x)$ are calculated since $W^{*}$ detects high valued ratios. Thus, $W$ will detect the dark shirt of the left person and $W^{*}$ will detect the white shirt of the right person in image Hall 190 [see Fig. 11(e) and (f), respectively].

\section{Discussion of RESUlts AND PERFORMANCE ASSESSMENT}

\section{A. Threshold Considerations}

- SM, DM, SCD: The thresholds for the state-of the art methods were chosen in accordance with the suggestions of the respective authors. Table 11 gives an overview of the thresholds applied. For the SM and DM, we tried thresholds of 0.01 and 100, respectively, and even with these low thresholds it was not possible to detect the object introduced in the objective illumination change test [see Figs. 4(a) and 5(a)].

- LDD: The thresholds for the LDD are between 0.03-0.11. The choice between these thresholds does not depend strongly on the type of the application. For significant illumination change conditions, as in the case of the Corridor sequence, it should be around 0.1 . In most other cases, a threshold around 0.05 is good.

- WM: Compared to the LDD, the WM is even less dependent on the threshold [see Table 11]. Fig. 11(e) and (f) suggests that a threshold $T_{W M}$ and $T_{W^{*}}$ greater than zero to detect physical changes with $W$ and $W^{*}$. For the detection of physical changes, both calculations should be positive

$$
W, W^{*}>T_{W M}, T_{W^{*}}>0 .
$$

In fact, the change detection masks obtained by $W^{*}$ are best when a threshold $T_{W^{*}}=0.6$ is applied. This threshold corresponds to the threshold applied to the $W$ as given in Table 11 . Consequently $T_{W^{*}}=T_{W M}$ and all thresholds of $W$ are kept for $W^{*}$.

\section{B. Dimension of Vectors}

Increasing the vector dimension can improve change detection masks by filling in undetected areas in objects, e.g., for LDD (see Fig. 8) and noisy areas [32]. As explained in Section V-A, a window sliding over the images is the region of support for the vectors. The main drawback is that by increasing the vector dimensions, the computational complexity of the LDD and WM do increase as well. The LDD, for example, needs almost twice the time when calculated on a 5 instead of a 9-dimensional vector.

\section{Summary and Comparison of Results}

- SM: The Shading Model is a slow change detector. This is due to the heavy variance and mean calculations in the areas. Furthermore, the process of dividing each image in fixed blocks of areas of interest takes also a lot of time. The object boundaries are very coarse due to 


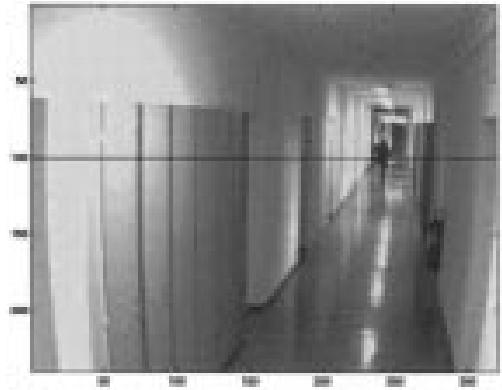

(a)

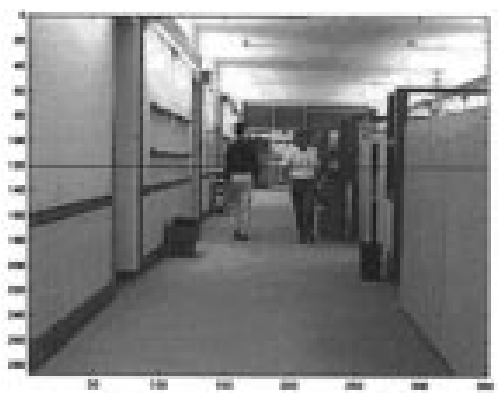

(a)

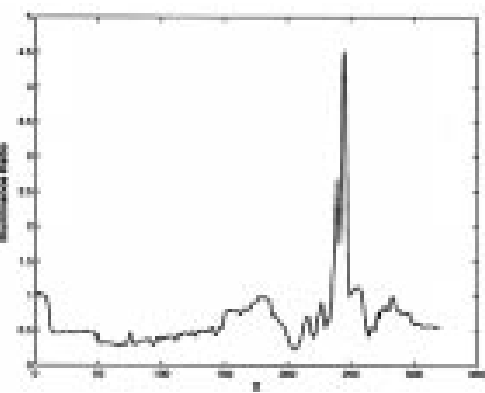

(b)

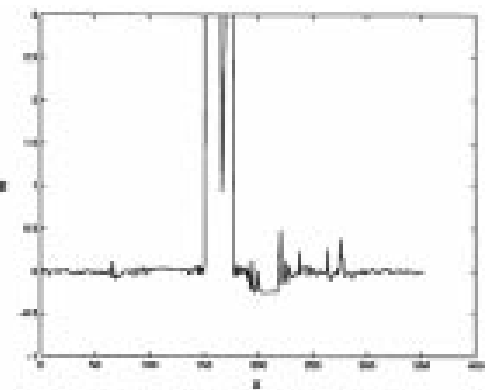

(b)

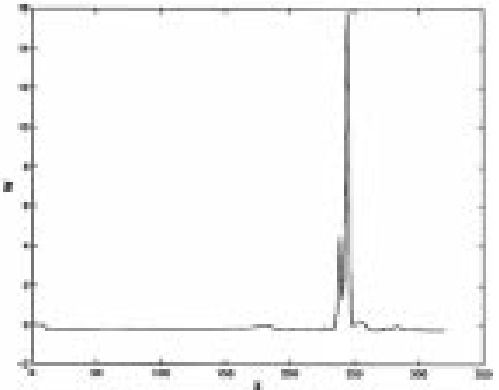

(c)

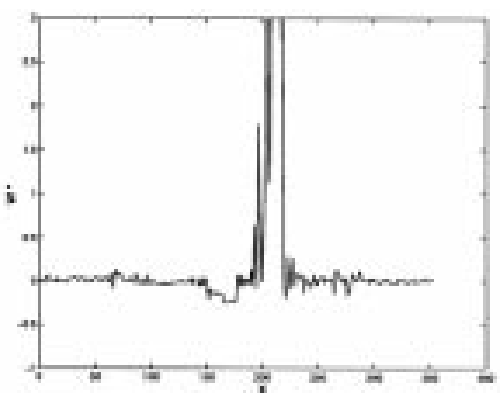

(c)

Fig. 11. Illuminance ratio of the 100th row of image Flur 221 (b) and (c) illustrates the Wronskian $W$ of the same row. (e) represents the Wronskian $W$ of the 120th raw of image Hall 190 and (f) the Wronskian $W^{*}$ for the same row of Hall 190.

Table 11

Table With Thresholds and Windows for the Change Detection Methods. In the Case of the LDD and WM, the Windows Are the Regions of Support for the Corresponding Vectors. The Windows Have Square Form. Therefore, a Size of 4 in This Table Means a $4 \times 4$ Windowing Applied to the Corresponding Sequences

\begin{tabular}{l||l|l|l|l|l}
\hline $\begin{array}{l}\text { Threshold I } \\
\text { Window }\end{array}$ & SM & DM & SCD & LDD & WM \\
\hline \hline $\begin{array}{l}\text { Obj. } \\
\text { Change }\end{array}$ & $0.06 \mid 4$ & $\begin{array}{l}300 \mid \\
3 \times 2\end{array}$ & $10^{-6} \mid 5$ & $0.05 \mid 5$ & $0.7 \mid 5$ \\
\hline Ill. Change & $0.05 \mid 4$ & $\begin{array}{l}300 \mid \\
3 \times 2\end{array}$ & $10^{-6}|5|$ & $0.05 \mid 5$ & $0.7 \mid 5$ \\
\hline Corridor & $0.05 \mid 4$ & $\begin{array}{l}300 \mid \\
3 \times 2\end{array}$ & $10^{-6}|5| 0.11 \mid 11$ & $0.5 \mid 5$ \\
\hline Room & $0.05 \mid 4$ & $\begin{array}{l}500 \mid \\
3 \times 2\end{array}$ & $10^{-6}|5| 0.03 \mid 7$ & $0.7 \mid 5$ \\
\hline Hall & $0.05 \mid 4$ & $\begin{array}{l}300 \mid \\
3 \times 2\end{array}$ & $10^{-6}|5| 0.08 \mid 5$ & $0.6 \mid 3$ \\
\hline Hand & $0.05 \mid 4$ & $300 \mid$ & $10^{-6}|5| 0.05 \mid 5$ & $0.7 \mid 3$ \\
& & $3 \times 2$ & & & \\
\hline
\end{tabular}

Table 12

Execution Times Per Image in Seconds for the Sequences Room and Corridor

\begin{tabular}{c|c|c|c|c|c}
\hline$i=$ & SM & DM & SCD & LDD & WM \\
\hline \hline O 1; Cor. & 6.35 & 0.75 & 3.17 & 2.14 & 1.36 \\
\hline O 1; Room & 6.35 & 0.72 & 2.73 & 2.14 & 1.36 \\
\hline G & 1 & 5 & 2 & 3 & 4 \\
\hline
\end{tabular}

the calculation of the Shading Model on square image areas. Object interiors are sometimes not detected since this method is variance based-the variance is zero for constant arguments (see Section V-E). This is the case in object interiors. Noise, diffuse reflections, and illumination changes are suppressed. The overall performance is $57 \%$ (see Table 13) of the total performance function given in (3). This results in a third place between all the tested change detectors.

- DM: The Derivative Model is very fast and detects contours, but in most cases these contours are not closed. It behaves more like an edge detector, due to the use of derivatives on the gray-value surface functions. These derivatives result in detection of high-gradient regions. As it is the case in the SM, noise, diffuse reflections, and illumination changes are removed. Therefore, third position with 48 performance is justified.

- SCD: The SCD approach is a good change detector provided there are no reflections or illumination changes present (see Fig. 6 Hand). Unfortunately, this is not the case in most real-world applications where there are always reflections and illumination changes. This pushes the SCD down to fifth place with a score of $35 \%$. Recently, the authors of this method have published a method rendering the SCD illumination invariant [27].

- LDD: The LDD is a fast approach. The object detection capabilities are good but there are problems with object interiors that are due to the nature of the variance (see Section V-E) (as explained for the SM). Nevertheless, the interior detection can be improved when the dimensions of the vectors is increased [32]. The object detection performance is actually close to the one of the SM. Although it is illumination invariant, the information on changed illumination conditions is not lost since it is present in the illuminance ratio's $k$ [see Fig. 11(b)]. So, if one needs 
Table 13

Performance Values According (1) and (3). The Right

Column Gives the Highest Possible Performance Value for

Each Assessment Requirements $p_{\max }$

\begin{tabular}{l||l|l|l|l|l||l}
\hline & SM & DM & SCD & LDD & WM & $p_{\text {max }}$ \\
\hline \hline$p_{O 1}$ & 5 & 25 & 10 & 15 & 20 & 25 \\
\hline$p_{O 2}$ & 5 & 5 & 15 & 10 & 15 & 15 \\
\hline$p_{O 3}$ & 0 & 0 & 0 & 0 & 15 & 15 \\
\hline \hline$p_{S 1}$ Cor. & 15 & 5 & 0 & 15 & 20 & 20 \\
\hline$p_{S 1}$ Room & 10 & 5 & 5 & 15 & 20 & 20 \\
\hline$p_{S 1}$ Hall & 10 & 10 & 15 & 15 & 15 & 20 \\
\hline$p_{S 1}$ Hand & 15 & 10 & 20 & 20 & 20 & 20 \\
\hline \hline$p_{S 2}$ Cor. & 15 & 10 & 0 & 15 & 15 & 20 \\
\hline \hline$p_{S 3}$ Cor. & 12 & 12 & 0 & 12 & 12 & 16 \\
\hline$p_{S 3}$ Room & 12 & 12 & 0 & 12 & 12 & 16 \\
\hline$p_{S 3}$ Hall & 12 & 12 & 0 & 12 & 12 & 16 \\
\hline \hline$p_{S 4}$ Cor. & 2 & 0 & 0 & 4 & 6 & 8 \\
\hline$p_{S 4}$ Room & 2 & 2 & 2 & 6 & 6 & 8 \\
\hline$p_{S 4}$ Hall & 2 & 4 & 6 & 4 & 4 & 8 \\
\hline$p_{S 4}$ Hand & 4 & 2 & 8 & 6 & 8 & 8 \\
\hline \hline$p_{S 5}$ Room & 4 & 4 & 2 & 4 & 8 & 8 \\
\hline$p_{S 5}$ Hall & 2 & 2 & 4 & 4 & 4 & 8 \\
\hline$P(E q .3)$ & 147 & 120 & 92 & 159 & 212 & 251 \\
\hline$P_{\%}$ & $57 \%$ & $48 \%$ & $35 \%$ & $67 \%$ & $84 \%$ & $100 \%$ \\
\hline & & & & & & \\
\hline & & & & \\
\hline
\end{tabular}

the illumination change information $k<1$ and the object change information $k>1$, LDD provides both of them. The overall performance is $67 \%$, which results in a second place between all the tested change detectors.

- WM: This approach is also very fast and provides the best results in almost all objective and subjective assessment requirements: the boundaries are closed, object interiors are detected, illumination invariance and noise reduction is provided. Furthermore, moving curtains are almost removed as well. Therefore, the first position with 84 performance level is merited. In case one wants to update the reference image to which the incoming current images are compared, the cases with $W=0$ and $W^{*}=0$ provide this possibility. This condition corresponds to unchanged regions in the current frame (i.e., background) and it indicates that the corresponding vectors are linearly dependent (26).

- Computational Complexity: Table 12 gives the computational complexity for the sequences Room and Corridor. It can be seen that with the exception of the SCD the algorithms showed almost no image content dependence.

\section{CONCLUSION}

The vector model of images introduced enabled us to incorporate useful theorems from linear algebra into a change detection scheme. In particular the application of the concept of linear dependence and independence is a powerful tool in this context. In the change detector formulation process, the constraint given from photometry were taken into consideration, i.e., between a pair of images the ratio of illuminances is an unambiguously measurable physical quantity. Change detectors based on the concept of linear dependence are the LDD and the WM.

The WM is robust against noise and it provides good change detection results, i.e., it detects objects and suppresses illumination changes successfully. Furthermore, changing environmental conditions such as changing cloud cover or moving trees in the background do not pose a significant problem to the WM.

The evaluation procedure for the three state-of-the art change detectors and the proposed two change detectors, i.e., LDD and WM, proved that the WM was the best performing change detector.

\section{ACKNOWLEDGMENT}

The authors would like to acknowledge the suggestions and the help of J. Tinsman and A. Dar.

\section{REFERENCES}

[1] C. S. Regazzoni and L. Marcenaro, "Object detection and tracking in distributed surveillance systems using multiple cameras," in NATO Advanced Studies Institute on Multisensor Data Fusion, Pitlochry, Perthshire, Scotland, June 25, 2000.

[2] G. L. Foresti, "Object recognition and tracking for remote video surveillance," IEEE Trans. Circuits Syst. Video Technol., vol. 9, pp. 1045-1062, Oct. 1999.

[3] R. T. Collins, A. J. Lipton, and T. K. Kanade et al., "Special section on video surveillance," Pattern Anal. Mach. Intell., vol. 22, Aug. 2000.

[4] Y. Raja et al., "Tracking and segmentation people in varying lighting conditions using color," in IEEE Proc. FG'98, Nara, Japan, Apr. $14-16,1998$

[5] L. Bedat et al., "Low-bit rate codec based on LAR method for videosurveillance via internet," in EUSIPCO 2000, Finland, Sept. 2000.

[6] C. S. Regazzoni, C. Sacchi, and C. Dambra, "Remote cable- based video surveillance applications: The AVS-RIO project," in Proc. ICIAP99, Venice, Italy, Sept. 27-29, 1999.

[7] B. T. Phong, "Illumination for computer generated pictures," Commun. ACM, vol. 18, pp. 311-317, 1975.

[8] K. Skifstad and R. Jain, "Illumination independent change detection for real world image sequences," CVIP, vol. 46, no. 3, pp. 387-399, 1989.

[9] Y. Z. Hsu et al., "New likelihood test methods for change detection in image sequences," Comput. Vis. Graph. Image Process., vol. 26, pp. 73-106, 1984.

[10] T. Aach et al., "Statistical model-based change detection in moving video," Signal Process., vol. 31, pp. 165-180, 1993.

[11] P. Villegas, X. Marichal, and A. Salcedo, "Objective evaluation of segmentation masks in video sequences," in WIAMIS'99 Workshop, Berlin, Germany, May 1999.

[12] M. Wollborn and R. Mech, "Procedure for objective evaluation of VOP generation algorithms,", Fribourg, ISO/IEC JTC1/SC29/WG MPEG97/2704, 1997.

[13] K. McKoen, R. Navarro-Prieto, B. Duc, E. Durucan, F. Ziliani, and T. Ebrahimi, "Evaluation of video segmentation methods for surveillance applications," in EUSIPCO 2000, Tampere, Finland, Sept. 2000.

[14] J. W. S. Liu, Real-Time Systems. Englewood Cliffs, NJ: PrenticeHall, 2000.

[15] B. Jaehne, Digital Image Processing, 4th ed. New York: SpringerVerlag, 1997.

[16] M. Born et al., Principles of Optics, 7th ed. London, U.K.: Cambridge Univ. Press, 1999.

[17] G. Wyszecki and W. Styles, Color Science: Concepts and Methods, Quantitative Data and Formulae Wiley, New York, 1982. 
[18] D. Koller et al., "Robust multiple car tracking with occlusion reasoning," Univ. California, Berkeley, CA, 94720.

[19] R. Jain, "Difference and accumulative difference pictures in dynamic scene analysis," Image Vis. Comput., vol. 2, pp. 99-107, 1984.

[20] M. Tekalp, Digital Video Processing. Englewood Cliffs, NJ: Prentice-Hall, 1995.

[21] R. Jain, D. Millitzer, and H. H. Nagel, "Separating nonstationary from stationary scene components in a sequence of real world TV-images," in Proc. 5th Int. Joint Conf. Artificial Intelligence, 1977.

[22] T. Aach and V. Metzler, "Bayesian algorithms for adaptive change detection in image sequences using Markov random fields," Signal Process. Image Commun., vol. 7, no. 2, 1995.

[23] S. Lui et al., "Statistical change detection with moments under timevarying illumination," IEEE Trans. Image Processing, vol. 7, Sept. 1998.

[24] L. Zeng et al., "Extracting illumination invariant face representation," in Machine Graphics \& Vision, Proc. 4th Conf. GKPO, Machocice, Poland, 1996.

[25] R. Mech et al., "2D shape estimation for moving objects considering a moving camera and cast shadows," in SPIE Conf. Visual Communication and Image Processing, San Jose, CA, 1999.

[26] A. V. Oppenheim, R. W. Schafer, and T. G. Stockham Jr, "Nonlinear filtering of multiplied and convolved signals," Proc. IEEE, vol. 56, pp. 1264-1291, Aug. 1968.

[27] D. Toth, T. Aach, and V. Metzler, "Bayesian spatio-temporal motion detection under time varying illumination," in EUSIPCO 2000, Finland, Sept. 2000.

[28] C. Enrique et al., Orthogonal Sets and Polar Methods in Linear Algebra. New York: Wiley, 1999.

[29] E. Durcan and T. Ebrahimi, "Improved linear dependence and vector model for illumination invariant change detection," in Real-Time Imaging, SPIE's Photonics West 2001-Electronic Imaging Conf., San Jose, CA, Jan. 21-26, 2001.

[30] H. Anton and C. Rorres, Elementary Linear Algebra. New York: Wiley, 1994
[31] M. Tenenbaum and H. Pollard, Ordinary Differential Equations. New York: Dover, 1963.

[32] E. Durucan and T. Ebrahimi, "Robust and illumination invariant change detection based on linear dependence for surveillance application," in Proc. 10th Eur. Signal Processing Conf. (EUSIPCO-2000), vol. 5, Tampere, Finland, Sept. 5-8, 2000, pp 1141-1144.

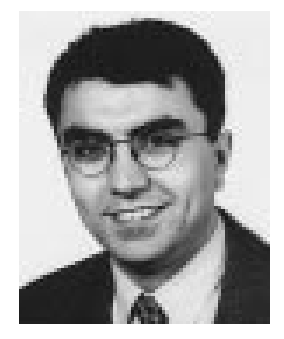

Emrullah Durucan graduated from the University of Bielefeld, Germany. He also studied at the Universities of Lyon, France, and Birmingham, U.K. He received the M.Sc. degree in particle physics from the Max-Planck-Institute (MPIK), Heidelberg.

He worked at CERN, Switzerland and FERMILAB, USA. In 1997 he joined the Signal Processing Laboratory, Swiss Federal Institute of Technology, Lausanne, Switzerland, where he is pursuing research toward a Ph.D degree.

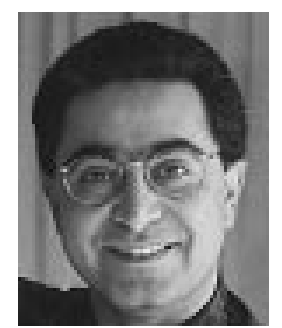

Touradj Ebrahimi (Member, IEEE) is currently Professor at the Signal Processing Laboratory, Swiss Federal Institute of Technology, Lausanne, Switzerland, where he is involved in research and teaching topics related to visual information processing. His research interests are image coding, segmentation and interpretation, security, and information theory. He is the author or co-author of more than 80 research publications and 10 patents.

Prof. Ebrahimi is a Member of SPIE. 